\title{
VALIDACIÓN DE PROTOTIPO SECADOR SOLAR INDIRECTO PARA EL DESHIDRATADO DE PAPAYA HAWAIANA (VARIEDAD SOLO SUNRISE)
}

\section{INDIRECT SOLAR DRYER PROTOTYPE VALIDATION FOR THE HAWAIIAN PAPAYA DEHYDRATE (ONLY SUNRISE VARIETY)}

\author{
Josseling Azucena Sobalvarro. ${ }^{1}$ \\ Azdiel Antonio Duarte Rodríguez ${ }^{1}$ \\ Neysis Ivania Gómez Chavarría ${ }^{1}$ \\ Karla Elizabeth Dávila. ${ }^{2}$
}

(recibido/received: 12-Septiembre-2017; aceptado/accepted: 5-Diciembre-2017)

RESUMEN: Esta investigación estuvo centrada en la validación de un prototipo de secador solar para el deshidratado de frutas, siendo el estudio de tipo experimental, ya que partió de la realización de pruebas de laboratorio para la caracterización de la materia prima utilizada "Papaya hawaiana variedad solo sunrise", así mismo se incluyó el diseño y construcción del secador solar, el cual se validó mediante el desarrollo de un tipo de producto, en este caso papaya deshidratada, para esto se tomaron en cuenta el control de las variables: tiempo, temperatura y peso de la muestra. Durante el desarrollo de la investigación se determinaron los valores óptimos para el tipo de corte ( juliana de $11 \mathrm{~mm}$ para el secado industrial y $8 \mathrm{~mm}$ para el secado solar), tiempo de deshidratado (4-5 horas secado industrial y 26-30 hrs secado solar), el tratamiento (sumersión de la muestra en ácido ascórbico previo al proceso de deshidratado) y las temperaturas de (40 ${ }^{\circ} \mathrm{C}$ para el industrial y $29-51^{\circ} \mathrm{C}$ para el solar), así como también los promedios de los parámetros físico químicos evaluados en la fruta: ${ }^{\circ}$ Brix (8.78), $\mathrm{pH}(6.57)$, porcentaje de humedad (90.62\%), resistencia $\left(5.72 \mathrm{~kg} / \mathrm{cm}^{2}\right)$ y madurez ( $80 \%$ maduro- $20 \%$ verde). Así mismo para la construcción del secador solar se determinó que el material más viable fue el metal ya que éste asegura una alargada vida útil de la tecnología, así como la calidad y la inocuidad de los alimentos.

PALABRAS CLAVE: Secador solar, secador industrial, temperatura, humedad relativa, características organolépticas

ABSTRACT: This research was focused on the validation of a solar dryer prototype for dehydration fruit, being the experimental study type, since it started with laboratory tests for the characterization of the raw material used "Papaya Hawaiian only sunrise variety", also included the design and construction of the solar dryer, which was validated through the development of a

\footnotetext{
${ }^{1}$ Ingeniera Agroindustrial. Graduada en la Universidad Nacional de Ingeniería, Sede Regional del Norte (UNI-RUACS) Nicaragua. josselingsovalbarro28@gmail.com,

2 Ingeniera Química. Docente Universidad Católica del Trópico Seco (UACTSE), Nicaragua..2008@yahoo.com
} 
type of product, in this case dehydrated papaya, for this the control of the variables was taken into account: time, temperature and weight of the sample. During the development of the research the optimal values for the type of cut were determined (julienne $11 \mathrm{~mm}$ for industrial drying and $8 \mathrm{~mm}$ for solar drying), dehydration time (4-5 hours industrial drying and 26-30 hrs drying solar), the treatment (submersion of the sample in ascorbic acid prior to the dehydration process) and the temperatures of $\left(40{ }^{\circ} \mathrm{C}\right.$ for the industrial and $29-51{ }^{\circ} \mathrm{C}$ for the solar), as well as the averages of the physical parameters chemicals evaluated in the fruit: ${ }^{\circ} \mathrm{Brix}(8.78), \mathrm{pH}(6.57)$, humidity percentage $(90.62 \%)$, resistance $\left(5.72 \mathrm{~kg} / \mathrm{cm}^{2}\right)$ and maturity $(80 \%$ mature- $20 \%$ green). Likewise, for the construction of the solar dryer, it was determined that the most viable material was the metal since it ensures an extended useful life of the technology, as well as the quality and safety of the food.

KEYWORDS: Solar dryer, industrial dryer, temperature, relative humidity, organoleptic characteristics.

\section{INTRODUCCIÓN}

El cultivo de frutas y hortalizas en Nicaragua tiene una fuerte contribución económica para los pequeños agricultores del país. Actualmente, Nicaragua representa un potencial fuerte para la producción frutícola de diferentes especies tropicales, autóctonas y comerciales, las cuales en su mayoría se pierden por causa de bajos niveles tecnológicos, accesos financieros y mercados; por tal razón es importante que se pongan en marcha nuevas alternativas ecológicas que permitieran la conservación de las frutas y la implementación de tecnologías que ayuden al agricultor con las frutas que no logran ser vendidas pero pueden ser aprovechadas de otra manera.

Así mismo, con el pasar del tiempo en diversos países se han realizado diversas investigaciones con el objetivo de buscar alternativas para aprovechar los recursos existentes mediante, la implementación de energías limpias y renovables, usando secadores solares que resultan ser útiles, eficientes y económicos para el deshidratado de los alimentos.

En correspondencia con lo anteriormente planteado, la investigación se basó en la validación de un prototipo de secador solar para la deshidratación de papaya hawaiana (variedad Solo Sunrise), a través de convección natural, que ayude a la conservación de las frutas, mantenga sus características organolépticas y a su vez sea amigable con el medio ambiente.

\section{METODOLOGÍA}

La investigación realizada fue de tipo experimental, con el diseño pre-experimento del tipo estudio de caso con una sola medición el cual fue tomado de Sampieri, Collado, \& Baptista (2010). Este consistió en determinar y aplicar el tratamiento con el cual se obtuvieron mejores resultados en el proceso productivo, en este caso el prototipo de secador solar, posteriormente se aplicó la medición de variables (temperatura y \% de humedad del producto), para observar el nivel de desarrollo y aceptación del producto. 
Las variables usadas fueron temperatura (variable independiente) y el \% de humedad del producto (variable dependiente), cabe señalar que en el prototipo de secador solar no hubo manipulación de la variable independiente, debido a que no se tuvo control de la misma, ya que, ésta estuvo en función de las condiciones climáticas existentes durante la etapa de experimentación.

Los experimentos realizados fueron:

- Comparación entre el prototipo de secador solar y el industrial (testigo), con muestras preparadas en igualdad de condiciones.

- Pruebas de capacidad máxima en el prototipo de secador solar.

En este sentido el desarrollo de esta investigación dio inicio con la caracterización de la materia prima, en la cual se hizo una evaluación de las características físico químicas de la materia prima, en este caso: ${ }^{\circ}$ Brix, \% humedad, resistencia, y madurez. Posteriormente se procedió con la aplicación de una serie de tratamientos hasta obtener el proceso productivo que permitiera la obtención de un producto final con características organolépticas aceptables, dichos tratamientos fueron: definición de corte y grosor, sumersión de la fruta en jarabe de azúcar, sumersión de la fruta en concentraciones de limón y sumersión de la fruta en solución de ácido ascórbico.

En relación a los parámetros de diseño, se determinaron mediante la búsqueda y recopilación de información de carácter primario y secundario, esto mediante la consulta en libros y sitios web, así como también la aplicación de entrevistas en lugares donde se han diseñado y construido tecnologías solares, de esta manera se determinó el diseño a construir. Posteriormente se realizó una evaluación de tres tipos de materiales de construcción (madera, plástico y metal) con el diseño anteriormente establecido en función de los costos, vida útil y calidad e inocuidad alimentaria, una vez determinado el material óptimo para el secador se procedió a su construcción.

Por otra parte, para realizar las curvas de secado se desarrollaron dos experimentos; el primero consistió en colocar 10 muestras en la tecnología solar e industrial, con el fin de obtener los tiempos de operación y el segundo experimento se desarrolló con el proceso productivo a capacidad máxima en el secador solar.

Posteriormente, se procedió a la evaluación de las características organolépticas del producto final obtenido en ambas tecnologías, para ello, se utilizó un panel evaluador compuesto por 10 personas, donde compararon ambas muestras obtenidas mediante secado solar e industrial.

Por el último, se calcularon y compararon los costos de producción a escala de laboratorio para el secado solar e industrial. 


\section{RESULTADOS Y DISCUSIÓN}

Los porcentajes obtenidos en las características físico químicas de la papaya como materia prima a utilizar, se muestran en la tabla 1 y en las figuras 1, 2 y 3 los tipos de cortes de la papaya.

Tabla 1: Resultados características físico químicas

\begin{tabular}{c|c}
\hline Característica & $\%($ Porcentaje) \\
\hline${ }^{\circ}$ Brix & 8.78 \\
\hline $\mathrm{pH}$ & 6.57 \\
\hline$\%$ humedad & 90.62 \\
\hline resistencia & $5.72 \mathrm{~kg} / \mathrm{cm}^{2}$ \\
\hline madurez & $80 \%$ maduro- $20 \%$ verde \\
\hline
\end{tabular}

Fuente: Elaboración propia

Figura 1: Papaya en corte "rodajas"

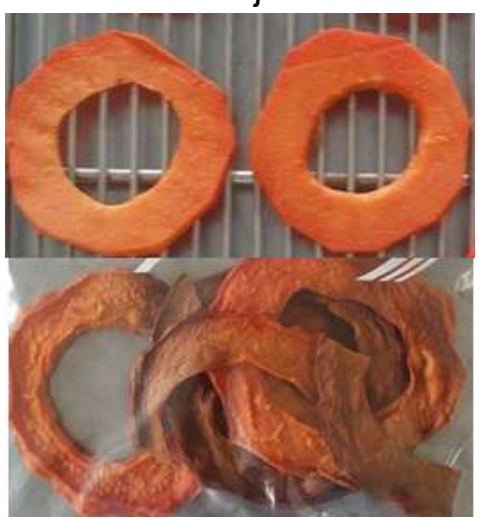

Figura 2: Papaya en corte "Cubos"

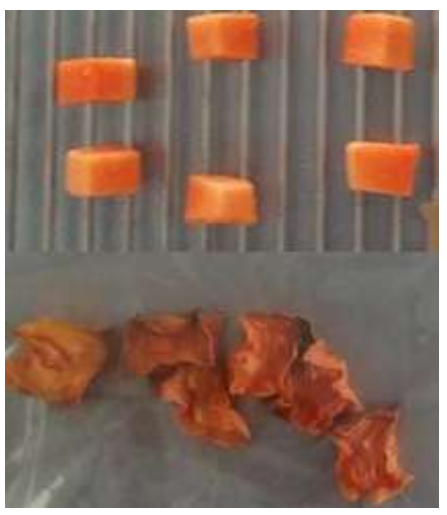

Fuente: Propia
Figura 3: Papaya en corte "juliana"

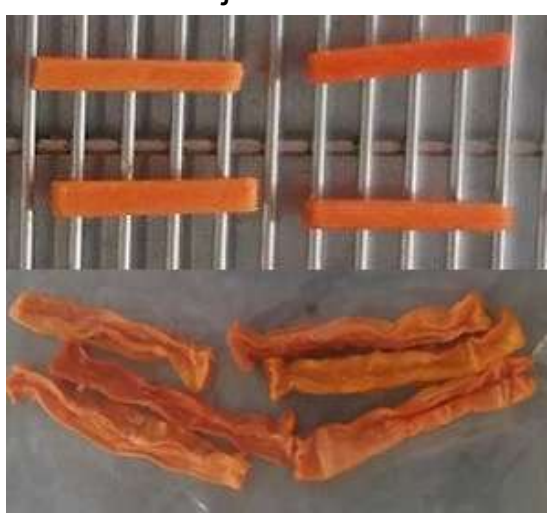




\begin{tabular}{|c|c|}
\hline \multicolumn{2}{|l|}{ Papaya sin ninguna sumersión } \\
\hline \multirow{3}{*}{ Sumersión de la papaya en jarabe de azúcar } & $25^{\circ}$ Brix \\
\hline & $50{ }^{\circ}$ Brix \\
\hline & $75^{\circ}$ Brix \\
\hline \multirow{2}{*}{$\begin{array}{l}\text { Sumersión de la papaya en concentraciones de } \\
\text { limón }\end{array}$} & $\begin{array}{l}125 \mathrm{ml} \text { en } 1 \text { litro de } \\
\text { agua. }\end{array}$ \\
\hline & $\begin{array}{l}250 \mathrm{ml} \text { en } 1 \text { litro de } \\
\text { agua. }\end{array}$ \\
\hline \multirow{3}{*}{$\begin{array}{l}\text { Sumersión de la fruta en concentraciones de } \\
\text { ácido ascórbico }\end{array}$} & $0.2 \%$ \\
\hline & $0.3 \%$ \\
\hline & $0.4 \%$ \\
\hline
\end{tabular}

Fuente: Elaboración propia

En cuanto al diseño y construcción de la tecnología, se obtuvo que éste sería un prototipo de secador solar indirecto, el cual fue construido con metal, vidrio $(5 \mathrm{~mm})$ y fibra de vidrio, lo cual puede ser observado en la figura 4.

Figura 4: Prototipo de secador solar indirecto con las mejoras aplicadas

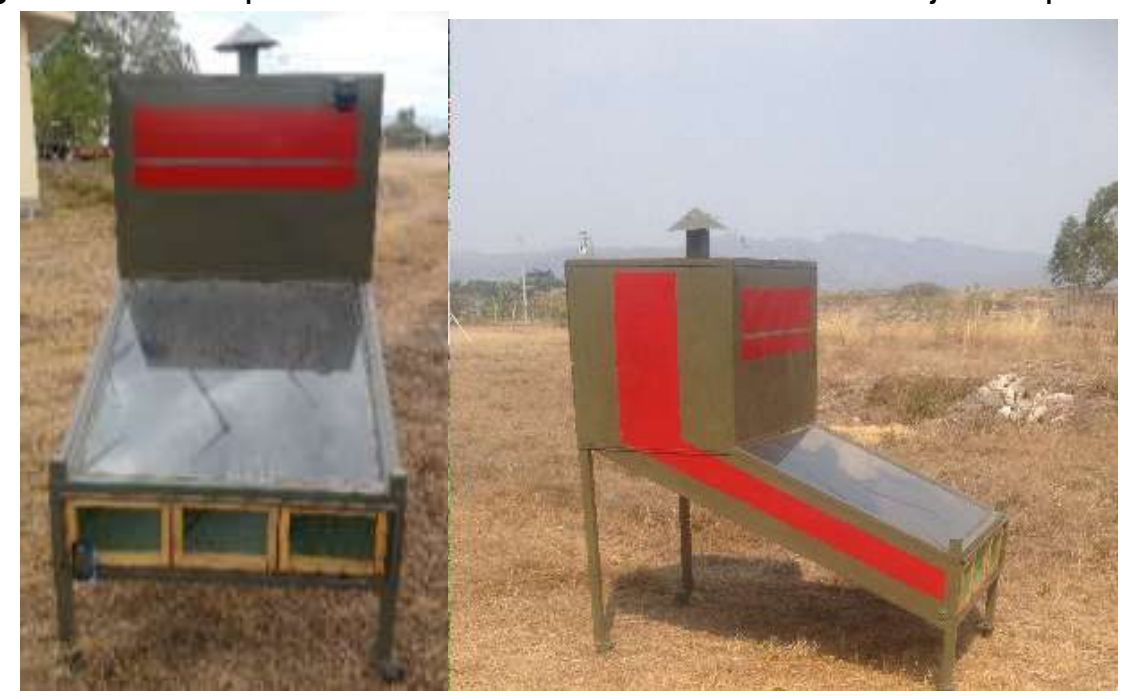

Fuente: Propia

Así mismo el promedio de capacidad de materia prima que puede ingresar al inicio de cada proceso es de $2.134 \mathrm{~kg}$. Es notorio destacar que en la etapa inicial de evaluación no se obtuvieron los resultados esperados en cuanto a las características organolépticas obtenidas en el producto y las temperaturas alcanzadas, por lo tanto se realizaron una serie de modificaciones las cuales consistieron en la incorporación de canaletas al colector solar, la reubicación de la chimenea y el cambio de compuertas y bandejas, obteniéndose posteriormente un incremento de la temperatura de $6{ }^{\circ} \mathrm{C}\left(42^{\circ} \mathrm{C}\right.$ a $\left.48^{\circ} \mathrm{C}\right)$ y la reducción del tiempo de secado de 7 a 3 días, cabe señalar que el grosor de papaya con el que se obtuvieron mejores resultados fue el corte juliana con grosor de $8 \mathrm{~mm}$. 
Por otra parte, como resultado de los dos experimentos ejecutados (pruebas de comparación entre el secador solar e industrial y pruebas de capacidad máxima en el secador solar), se obtuvo como tiempos de operación para el secador solar de 26 a 30 horas y de 4 a 5 horas en la tecnología industrial, de igual forma se conoció la velocidad de secado la cual fue de $5.02 \%$ /horas en el secado solar y $15.35 \%$ /hora para el secado industrial. Luego, para el segundo experimento se obtuvo un tiempo de secado de 51 a 52 horas y la temperatura máxima alcanzada fue de $53^{\circ} \mathrm{C}$, esto se puede notar en la figura 5.

Figura 5: Temperatura máxima alcanzada en secador solar

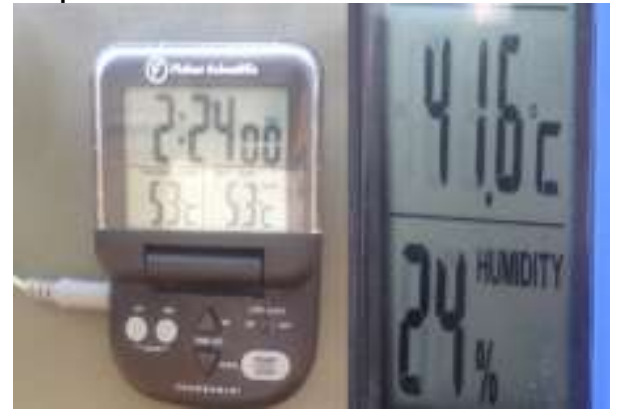

Fuente: Propia

En la evaluación de las características organolépticas del producto final obtenido en ambas tecnologías, se conoció que la muestra proveniente de secador solar obtuvo mejor aceptación, en relación a la apariencia $(10 / 10)$ y textura $(7 / 10)$, por otra parte, en cuanto al sabor y preferencia ambas muestras obtuvieron valores iguales (5/10). En este sentido las diferencias anteriormente descritas entre ambas muestras se pueden observar en la figura 6 y 7 .

Figura 6: Comparación de muestras secado solar e industrial

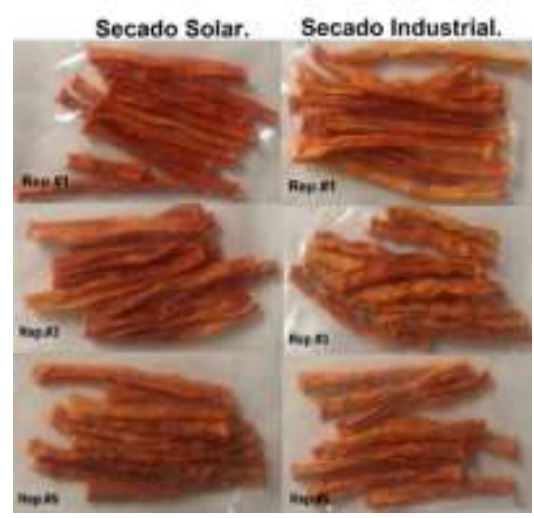

Figura 7: Muestras de prueba de capacidad máxima en secador solar

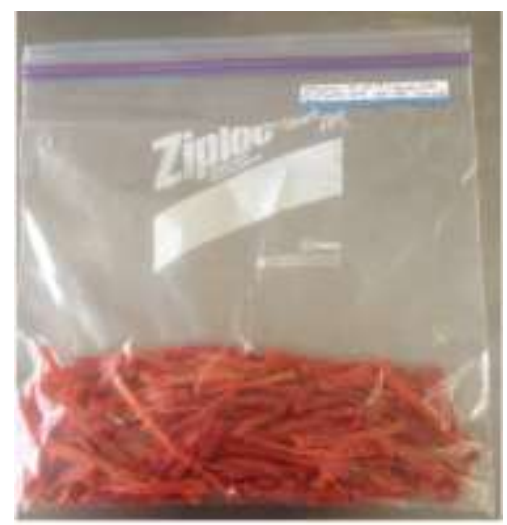

Por el último, para los costos de producción a escala de laboratorio para el secado solar e industrial, se obtuvo que el costo por gramo de producto final fue de $\mathrm{C} \$ 2.63$ para la tecnología solar y de $C \$ 1.99$ para la tecnología industrial. Así mismo, el costo de adquisición para el secador solar fue de, $C \$ 17,850.00$ y $C \$ 76,843.9$ para la tecnología industrial. 


\section{CONCLUSIONES}

Se determinó que los parámetros de caracterización en el secador industrial, fueron: la madurez (80\% maduro - $20 \%$ verde), las características físico químicas como: ${ }^{\circ}$ Brix (8.78), $\mathrm{PH}(6.57), \%$ humedad (90.62) y resistencia $\left(5.72 \mathrm{~kg} / \mathrm{cm}^{2}\right)$. En cuanto al tipo de corte donde se obtuvieron mejores resultados en la tecnología industrial, fue juliana con grosor de $11 \mathrm{~mm}$, sumergido en una solución $0.3 \%$ de ácido ascórbico.

En cuanto, a los parámetros de diseño del prototipo de secador se determinó que éste debe ser construido con acero comercial, vidrio $(4 \mathrm{~mm})$ y fibra de vidrio con una capacidad de 2.134 kilogramos distribuidos en dos bandejas de $0.69 \mathrm{~m} \times 0.45 \mathrm{~m}$. Con las modificaciones aplicadas (incorporación de canaletas al colector solar, reubicación de la chimenea, cambio de compuertas y bandejas), se logró un incremento de temperatura de $6^{\circ} \mathrm{C}$ y la reducción del tiempo de secado de 7 a 3 días.

Así mismo, en la comparación de la tecnología solar e industrial, se determinó, que el tiempo del proceso de deshidratado en la tecnología solar fue de 26 a 30 horas aproximadamente, alcanzando temperaturas en un rango de 29 a $51^{\circ} \mathrm{C}$, en cambio, en la industrial fue de 4 a 5 horas, manteniendo una temperatura constante $\left(40^{\circ} \mathrm{C}\right)$. Así mismo la velocidad de secado obtenido en la tecnología solar fue de $5.02 \%$ /hora y en la industrial de $15.35 \%$ /hora, no obstante, aunque la velocidad de secado sea menor en la tecnología solar, la pérdida de humedad en ésta, se dio con mayor uniformidad y las características organolépticas obtenidas fueron mejores, debido a que la intensidad de la temperatura es menor.

De igual manera, durante las pruebas a máxima capacidad para el secador solar, se alcanzaron las temperaturas más altas en la cámara de secado $\left(53^{\circ} \mathrm{C}\right)$, los tiempos de deshidratado fueron de 51 a 56 horas.

Por otra parte, al comparar las características organolépticas de las muestras de $8 \mathrm{~mm}$, procesadas en ambas tecnologías, los panelistas determinaron que la muestra con mejor apariencia fue la del secado solar, sin embargo, el $60 \%$ de los panelistas consideraron que la industrial tuvo mejor olor, ya que las obtenidas mediante el proceso solar no presentaron olor intenso en comparación con la industrial. En cuanto al sabor $50 \%$ de los panelistas indicaron que el mejor sabor lo aportaron las muestras de la tecnología solar y el $50 \%$ restante, las provenientes de la tecnología industrial, finalmente al evaluar la preferencia de las muestras 50 $\%$ panelistas se inclinaron a las muestras del solar y el otro $50 \%$ para el industrial.

Al comparar los costos de producción en gramos, de ambas tecnologías, resultó más barato producir en el industrial ( $C \$ 1.99$ ) que en el solar ( $C \$ 2.63)$, donde se obtuvo una diferencia de $\mathrm{C} \$ 0.64$, esto se debió a la cantidad de horas necesarias para desarrollar cada proceso, siendo mayor el tiempo para el secado solar. Por otra parte es importante destacar que el costo de adquisición de la tecnología solar resulta más económica ( $C \$ 17,850.00)$, que la industrial (C\$ 76,843.9), lo cual, la hace más accesible para los pequeños productores de la zona rural. 
Así mismo en la evaluación de los costos, se encontró que para productores del sector rural, resulta de mayor conveniencia adquirir la tecnología solar, debido a que la inversión inicial es mucho menor, así mismo, al comparar los costos de producción, éstos, resultan más accesibles en la tecnología solar. Por otra parte, es importante destacar que en muchos casos dichos productores no poseen acceso a energía eléctrica por lo que resulta más asequible el aprovechamiento de la energía solar.

\section{ABREVIATURAS}

mm: milímetros, gr: gramos, hrs: horas, ${ }^{\circ} \mathbf{C}$ : grados Celsius, pH: potencial hidrogeno

\section{REFERENCIAS}

Borda, M. (2013). El Proceso de Investigación: Visión general de desarrollo. Barranquilla, Colombia: Universidad del Norte.

MipymeDigital. (20 de marzo de 2012). Frutas Deshidratadas. Recuperado el 04 de Junio de 2016, de http://www.internovachile.com: http://www.internovachile.com/es frutas.php.

\section{AGRADECIMIENTOS}

Agradecemos a nuestra tutora MSc. Karla Elisabeth Dávila, por ser nuestro principal apoyo y brindarnos todo sus conocimientos y consejos, durante el desarrollo de este trabajo.

A nuestro maestro, Lic. Víctor Parrales, Q.P.E.D, un ejemplo de fuerza, inteligencia y perseverancia, quien siempre se desempeñó de manera exitosa en todas las asignaturas que nos impartió a lo largo de toda la carrera y quien nos apoyó durante este proyecto de investigación.

Al M. Sc. Claudio Pichardo, docente de la Universidad Nacional Agraria (UNA), MSc. Luís María Dicovskiy sub director de la Universidad Nacional de Ingeniería (UNI-RUACS) y M. Sc. Susan Kine directora del Grupo Fénix (centro de mujeres solares Togalpa- Nicaragua), por toda la orientación y apoyo técnico brindado para la ejecución de este estudio.

Al PNUD (Programa de Naciones Unidas para el Desarrollo) por brindarnos gran parte de la colaboración financiera para realizar esta investigación. 


\section{SEMBLANZA DE LOS AUTORES}
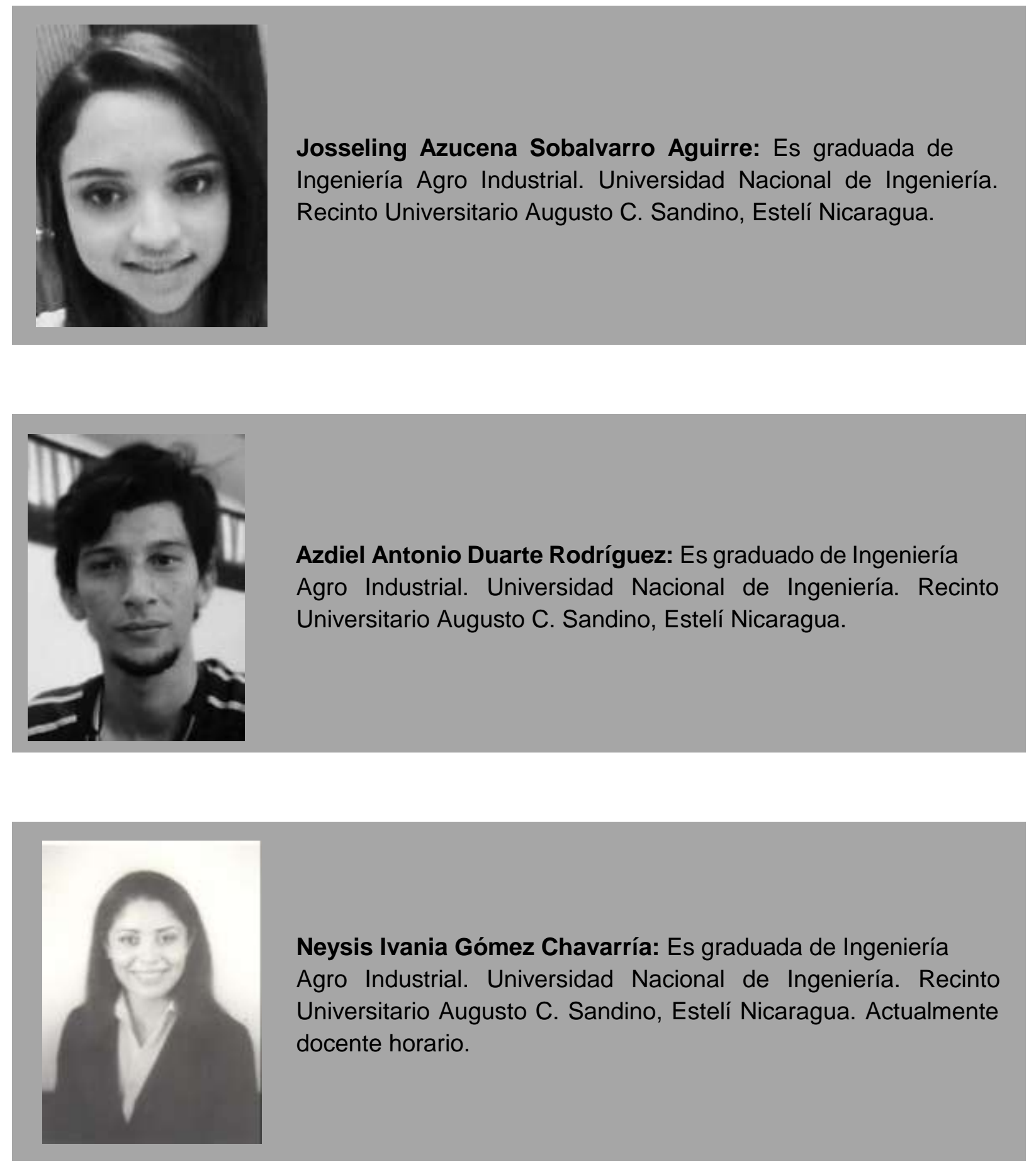